\title{
A Simple Photogrammetry Rig for the Reliable Creation of 3D Artifact Models in the Field
}

\section{Lithic Examples from the Early Upper Paleolithic Sequence of Les Cottés (France)}

\section{Samantha Thi Porter, Morgan Roussel, and Marie Soressi}

The ability to create three-dimensional (3D) models of artifacts has begun to revolutionize the way archaeologists document, analyze, and disseminate data about the objects they study. For example, lithic analysts have utilized 3D representations of artifacts in a myriad of ways. These investigations include, but are not limited to, studies of lithic fracture mechanics (Clarkson and Hiscock 2011;
Lin et al. 2013), measures of reduction (Clarkson 2013; Grosman et al. 2011; Lin et al. 2010; Morales et al. 2015), and the quantitative characterization of different reduction systems and tool types using methods such as vector analysis and geometric morphometrics (Bretzke and Conard 2012; Clarkson et al. 2006; Gingerich et al. 2014; Davis et al. 2015; Grosman et al. 2008; Riddle and Chazan 2014;

\section{ABSTRACT}

Three-dimensional (3D) artifact modeling is becoming an increasingly utilized tool in archaeology. In comparison with other methods of 3D scanning, photogrammetry has the benefits of being relatively inexpensive, mobile, and more adaptable for use in field conditions. As part of a larger project to document variability in lithic production systems across the Middle to Upper Paleolithic Transition in Western Europe, we developed a photography rig for the express purpose of systematically capturing images for the creation of 3D photogrammetric models. This low-cost rig greatly streamlines both the photo-taking and post-processing stages of model creation. Additional tips relating to the coating of difficult-to-capture objects with a mineral spray are also provided. Threedimensional models of lithic cores from the Châtelperronian, Protoaurignacian, and Early Aurignacian levels of the site of Les Cottés (France) are presented as examples of the quality of model that can be produced using this system.

Modelar artefactos en 3D se está convirtiendo en una de las herramientas más utilizadas en arqueología. En comparación con otros métodos de modelado en 3D, el registro de la fotogrametría tiene las ventajas de ser relativamente barato, móvil, y más adaptable para usar en condiciones de campo. Como parte de un proyecto para documentar la variabilidad en los sistemas de producción lítica a través de la transición del Paleolítico Medio al Paleolítico Superior en la Europa del Oeste, una plataforma de fotografía fue desarrollada con el propósito expreso de tomar fotografías para la creación de modelos fotogramétricos 3D. Esta plataforma de bajo coste agiliza en gran medida tanto la toma de fotografías como las etapas de post-procesamiento de la creación del modelo. También, se proporcionan consejos adicionales relativos al recubrimiento los objetos difíciles de capturar en un aerosol mineral. Los modelos 3D de los núcleos líticos de la Chatelperroniense, Protoauriñaciense, y los niveles del Auriñaciense antiguo del sítio de Les Cottés (Francia) se proporcionan como ejemplos de la calidad del modelo que se puede producir usando este sistema. 
Shott and Trail 2010). This is in addition to other benefits, such as the ability to create digital artifact archives and the capacity to openly disseminate archaeological datasets in new ways, including 3D printing (Means et al. 2013; Olson et al. 2014; Shott 2014).

That being said, 3D artifact documentation and the dissemination of 3D data still remain on the brink of becoming truly mainstream practices. This can partially be attributed to the costs traditionally associated with 3D scanning. These costs include both the money needed to purchase a 3D scanner and the time needed to learn how to utilize the requisite machinery and software properly. Most 3D scanners used in archaeological applications work by emitting some form of light at a target object and then using a camera to capture the way this light deforms (Blais 2004). These types of systems employ either lasers (e.g., the popular NextEngine scanner ${ }^{1}$ ) or patterns of white light (e.g., the Breuckmann ${ }^{2}$ or DAVID SLS-2 ${ }^{3}$ scanners).

In contrast, close-range photogrammetric modeling works by finding reference points on digital images taken from multiple angles and then determining the relative position of these points in 3D space via triangulation (Luhmann et al. 2013). Because photogrammetry software works directly off of digital images, the only equipment truly required is a digital camera. Photogrammetry software applications, such as the popular Agisoft PhotoScan, ${ }^{4}$ are relatively affordable, especially for educational users. Open source programs such as VisualSFM, ${ }^{5}$ in combination with PMVS2 ${ }^{6}$ and Meshlab, ${ }^{7}$ have been shown to be capable of producing comparable results (Kersten and Lindstaedt 2012). The free application 123D Catch $^{8}$ from Autodesk even makes it possible to create 3D models using only a smart phone, although this program is not recommended for professional use (Kersten and Lindstaedt 2012; McCarthy 2014).

Despite the potential of close-range photogrammetry, we find that while many people within the field of archaeology may be aware of the possibilities these new methods provide they do not necessarily have easy access to the appropriate resources they need in order to skillfully and consistently apply these methods themselves. In this paper, we aim to address this problem of practice in several ways. First, we describe a photography rig we have developed to create models of small objects (between approximately 5 and $15 \mathrm{~cm}$ ), and explain how this rig mitigates common problems inherent in close-range photogrammetry. Next, we outline our protocols for image capturing and processing. In order to demonstrate the quality of model that can be produced using this type of system, we present models of a series of lithic cores from the Paleolithic site of Les Cottés (France), which were created using the protocols outlined in this paper. Lithic artifacts can be extremely difficult to scan due to raw material properties such as shininess and translucence. We will show how the application of a talc-based product known as developer spray can greatly enhance the quality of 3D models of these types of artifacts. Next, we will briefly discuss differences between close-range photogrammetry, laser scanning, and structured light scanning. Finally, we discuss the utility of creating 3D objects with a specific focus on the field of lithic analysis and provide some thoughts on where these innovations may lead us in the near future.

\section{DEVELOPING AND USING A PHOTOGRAMMETRY RIG}

This project began approximately two years ago when the primary author decided it would be worthwhile to incorporate 3D data into her ongoing dissertation project. It was determined early on that it would not be possible to use either a laser or a structured light scanner for this purpose due to the associated costs, constraints on time, and the primary author's inability to transport a large amount of equipment to the number of locations necessary. Previous work has shown that photogrammetric models of lithic artifacts have the potential to be used for analysis, and that measurements made on these digital models have a low margin of error in comparison to measurements taken on the actual objects using dial calipers (Sumner and Riddle 2008). As a result, a photogrammetry-based solution was sought.

The decision to use photogrammetry for this project posed several problems in need of solutions. First of all, it is considered best practice in project photogrammetry for an object to remain stable. In other words, instead of rotating an object relative to the camera (as is normally done in laser and structured light scanning), it is ideal for a photographer to rotate around a target object. This is because one changes the object's relationship to environmental light sources by moving or rotating an object within an environment. This can, in turn, change the pattern of shadows cast on the object's surface, which can subsequently confuse the ability of photogrammetry software to find consistent reference points across images. Nevertheless, a rotating stage setup was decided on, since it saves substantial space, time, and effort on the part of the operator. Through experimentation, it was found that issues with shadows can be largely mitigated by simply flooding the target object with light from all angles visible to the camera.

The authors also determined that it would be best to use a backdrop behind the target object. Without a backdrop, photographs are likely to include extraneous elements, such as tabletops, architectural elements of the work space, etc. Agisoft PhotoScan allows users to apply what are known as image masks in order to isolate the target object from any background elements. The software ignores these masked elements during subsequent processing stages. As a result, masking has the benefit of speeding up the processing time. Masking also serves to more accurately define the limits of an object. This feature is especially important in creating models of lithic artifacts, which often have thin, sharp edges that are often difficult to capture using other scanning methods. Another advantage of using a backdrop is that masks can be created automatically. Detailed step-by-step instructions of how to accomplish this in two different ways are available as part of this article's supplemental materials (Supplemental Instructions 1).

\section{Rig Components}

The components listed below comprise the rig developed for this project (Figure 1). The authors encourage readers not to view this as a definitive list, but rather one example of elements 
TABLE 1. Cost of Equipment and Software Applications Mentioned in This Article.

\section{capital W}

capital

L, S

\begin{tabular}{|c|c|c|c|c|}
\hline \multicolumn{2}{|l|}{ Element } & Purpose & \multirow{2}{*}{$\begin{array}{c}\text { Additional Product Information } \\
\text { Cost reflects price for } 1 \mathrm{~m}\end{array}$} & \multirow{2}{*}{$\frac{\text { Approximate Cost }}{\$ 20}$} \\
\hline Black velvet & \multirow{2}{*}{ capital S } & Photo backdrop & & \\
\hline Foam tiles & & Backdrop support & Comes in a set of 12 tiles & $\$ 16$ \\
\hline \multicolumn{2}{|c|}{ Kitchen turntable / lazy șusan } & Used to rotate the object & & $\$ 13$ \\
\hline \multicolumn{2}{|c|}{ Coleman Quad LED lantern } & Object illumination & Max of 47.5 lumens per panel & $\$ 50$ \\
\hline \multicolumn{2}{|c|}{ Fulcrum LED clip-on task light } & Object illumination & Brightness not listed & $\$ 17$ \\
\hline \multirow{2}{*}{\multicolumn{2}{|c|}{$\begin{array}{l}\text { Grey kneadable Rubber Eraser } \\
\text { Laminated scale }\end{array}$}} & Gray kneadable rubber & & $\$ 6$ \\
\hline & & scam, privor angmmictre aid & Cost reflects price of lamination & $\$ 2$ \\
\hline \multicolumn{2}{|l|}{ wood round } & Homemade turntable & & $\$ 5$ \\
\hline \multicolumn{2}{|c|}{ tazy susan bearing } & Homemade turntable & & $\$ 6$ \\
\hline \multicolumn{2}{|c|}{ Camera tripod } & Camera support & & prices vary \\
\hline \multicolumn{2}{|c|}{ Cannon PowerShot SX260 } & Image capture & 12.1 megapixel sensor & (in 2013) \$300 \\
\hline \multicolumn{2}{|c|}{ Sony Cyber-Shot RX100i } & Image capture & 20.2 megapixel sensor & $\$ 400$ \\
\hline \multicolumn{2}{|c|}{$\begin{array}{l}\text { Agisoft PhotoScan Standard } \\
\text { Edition }\end{array}$} & Photogrammetry Software & Stand-Alone license & $\$ 179$ \\
\hline \multicolumn{2}{|c|}{$\begin{array}{l}\text { Agisoft PhotoScan Standard } \\
\text { Edition }\end{array}$} & Photogrammetry Software & Educational license & $\$ 59$ \\
\hline \multicolumn{2}{|c|}{$\begin{array}{l}\text { Agisoft PhotoScan Professional } \\
\text { Edition }\end{array}$} & Photogrammetry Software & Stand-Alone license & $\$ 3499$ \\
\hline \multicolumn{2}{|c|}{$\begin{array}{l}\text { Agisoft PhotoScan Professional } \\
\text { Edition }\end{array}$} & Photogrammetry Software & Educational license & $\$ 549$ \\
\hline \multicolumn{2}{|c|}{ Geomagic Design X } & 3D Mesh Editing Software & & price not publically listed \\
\hline \multicolumn{2}{|c|}{ VisualSFM } & Photogrammetry Software & http://ccwu.me/vsfm/ & (open source) \$0 \\
\hline \multicolumn{2}{|l|}{ CMVS } & Photogrammetry Software & http://www.di.ens.fr/cmvs/ & (open source) \$0 \\
\hline \multicolumn{2}{|l|}{ Meshlab } & 3D Mesh Editing Software & $\begin{array}{l}\text { http://meshlab.sourceforge. } \\
\text { net/ }\end{array}$ & (open source) \$0 \\
\hline \multicolumn{2}{|c|}{ NextEngine laser scanner } & 3D scanner + software & & $\$ 2995$ \\
\hline \multicolumn{2}{|c|}{$\begin{array}{l}\text { David-SLS-2 structured light } \\
\text { scanner }\end{array}$} & 3D scanner + software & & $\$ 2995$ \\
\hline
\end{tabular}

Note: Prices are in US Dollars and reflect amounts listed online for purchases within the United States at the time of final article submission. Free trials of Agisoft projects are available prior to purchase. .

$$
\text { PhotoScan }
$$

that have been proven to work well together as a coherent system. These components may be substituted or adjusted based on researchers' own needs and the materials available to them. The respective cost of all rig elements, as well as software packages discussed in this paper, is available in Table 1. We note that, although the rig discussed here was developed independently, a similar setup has previously been generally described by Olson et al. (2013), albeit in less detail.

- A kitchen turntable, also known as a lazy Susan, to serve as a rotating platform. This was spray-painted black, and increments of 10 degrees were marked around the exterior using a silver permanent marker. It is also possible to construct a turntable by affixing a lazy Susan bearing to a round cut of wood or a similar material. Because the quality of the bearings in inexpensive off-the-shelf turntables tends to be fairly low, we have found that self-constructed turntables tend to be more stable and to rotate more smoothly.

- Two pieces of black velvet, one larger piece to serve as a backdrop and a smaller circular piece to cover the top of the turntable.

- A set of three foam puzzle tiles. These are normally used as padding under exercise equipment or flooring in children's play areas. These were used to provide a structure on which to drape the velvet backdrop.

- A battery-powered LED light with a flexible neck and the ability to clip to the edge of a table. This light is used for overhead illumination.

- A means of illuminating objects from the front and sides. We used a Coleman Quad LED Lantern. This lantern consists 


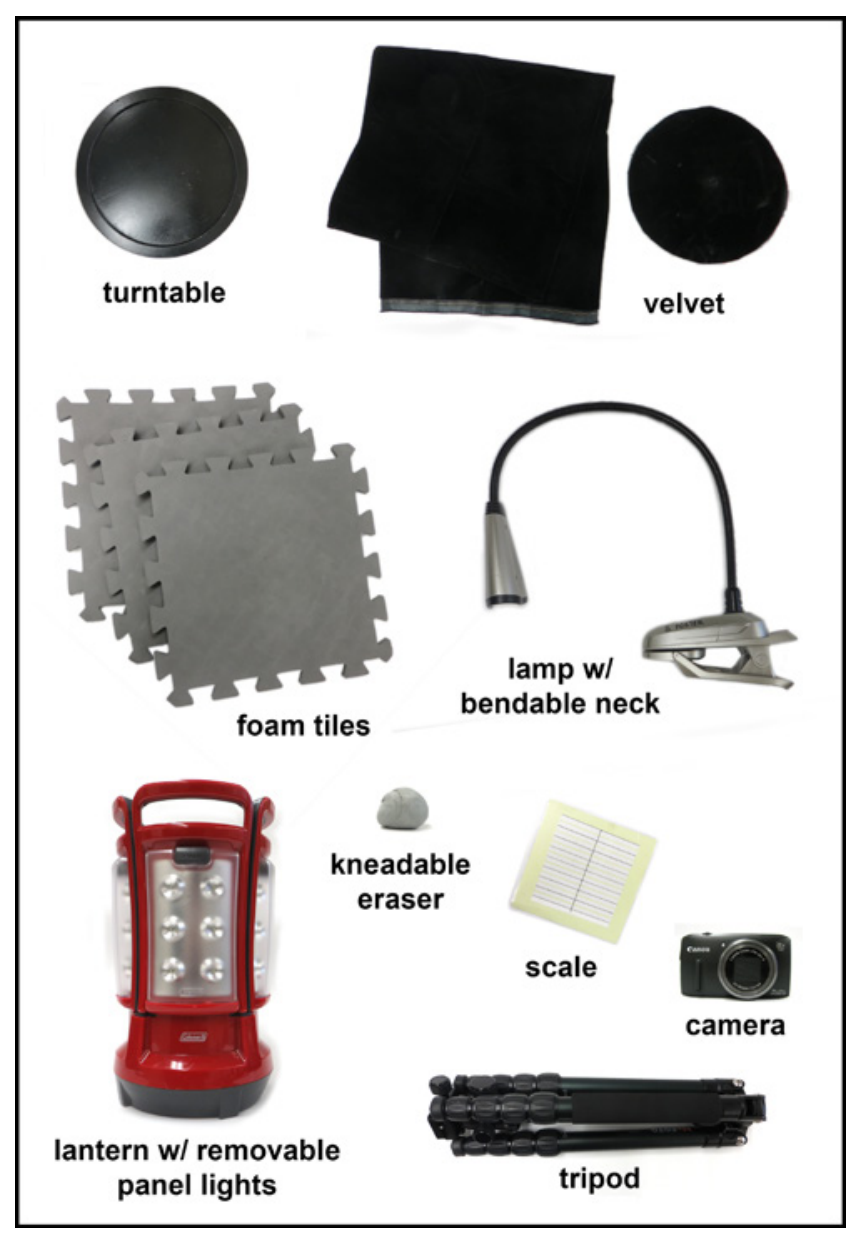

FIGURE 1. Materials used by the authors to create a portable photogrammetry rig.

of four LED panels, which can be detached from the main lantern base.

- A flat scale approximately $10 \mathrm{~cm}$ by $10 \mathrm{~cm}$ in size. This was made by laminating a square piece of $\mathrm{mm}$ paper strengthened with cardstock. Every $5 \mathrm{~mm}$ was highlighted using black or green marker to increase readability. We have since designed a scale specifically to facilitate image alignment and model scaling (Supplemental Scale 1).

- A means of supporting the objects being captured. In this case, a gray kneadable rubber eraser was used. If it can be avoided, objects with few faces, such as lithic flakes, should not be laid flat. Laying objects flat can make it difficult for software to reconstruct the morphology of the artifact's edges, as well as relationship between the two sides of the object. This supporting material can be edited out of the 3D model during processing (see Supplemental Instructions 2).

- A camera tripod.

- A digital camera. The camera used to produce the models for this paper was a compact 12.1 megapixel Cannon PowerShot SX260.

\section{RIG SETUP AND USE}

Here, we provide detailed instructions on how we set up and use our rig. We have established the following photography protocol in order to ensure that there are sufficient images, as well as sufficient overlap between images, to reliably reconstruct object geometry. A video demonstration of us using our rig to capture a set of object photographs can be accessed as part of this article's supplemental materials (Supplemental Video 1).

\section{Rig Setup Instructions}

1. Assemble three foam puzzle tiles in a corner configuration (Figure 2a).

2. Drape background fabric (e.g., black velvet) over the tiles.

3. Set down the turntable with the $0^{\circ}$ mark facing forward. If necessary, place a smaller piece of background material on the turntable's surface.

4. Position the camera on a tripod at approximately the same height as the turntable (Figure 2b).

5. Clip the flexible LED lamp to the table's edge and direct the head downward towards the turntable's center.

6. Place the LED panel lamps on both side of the camera and rotate them to face the turntable's center.

7. Place the scale on the center of the turntable and then position the object on top of the scale (Figure 2c).

8. If the object is not sufficiently illuminated from all sides, additional LED panel lights may be added (Figure 2d).

\section{Photography Instructions}

1. With the camera positioned at approximately the same height as the turntable, take a picture of the artifact's label or bag. This will make sorting images later much easier, especially if many objects are photographed in the same session.

2. Make sure the turntable is rotated so that the $0^{\circ}$ mark is facing the camera. Take the first picture.

3. Rotate the turntable $30^{\circ}$ so that the $30^{\circ}$ mark is facing the camera. Take a second image. Continue rotating the turntable and taking photographs every $30^{\circ}$ until the turntable has been fully rotated (i.e., at the $60^{\circ}, 90^{\circ}, 120^{\circ}, 150^{\circ}, 180^{\circ}$, $210^{\circ}, 240^{\circ}, 270^{\circ}, 300^{\circ}$, and $330^{\circ}$ marks). On our turntable, these intervals are annotated with a T-shaped symbol.

4. Using the tripod, raise the camera slightly and tilt it towards the target object. For small objects, we usually raise the camera approximately 5 to $10 \mathrm{~cm}$.

5. Rotate the turntable so that the $10^{\circ}$ mark is facing the camera. Take a photo. Rotate the turntable $30^{\circ}$ so that the $40^{\circ}$ mark is facing the camera. Take another photo. Continue rotating the turntable and taking photographs every $30^{\circ}$ until the turntable has been fully rotated (i.e., at the $70^{\circ}, 100^{\circ}$, $130^{\circ}, 160^{\circ}, 190^{\circ}, 220^{\circ}, 250^{\circ}, 280^{\circ}, 310^{\circ}$, and $340^{\circ}$ marks). On our turntable, these intervals are annotated with a $\uparrow$ symbol.

6. Raise the camera again and tilt it towards the target object. Rotate the turntable so that the $20^{\circ}$ mark is facing in the direction of the camera. Take a photo. Rotate the turntable 


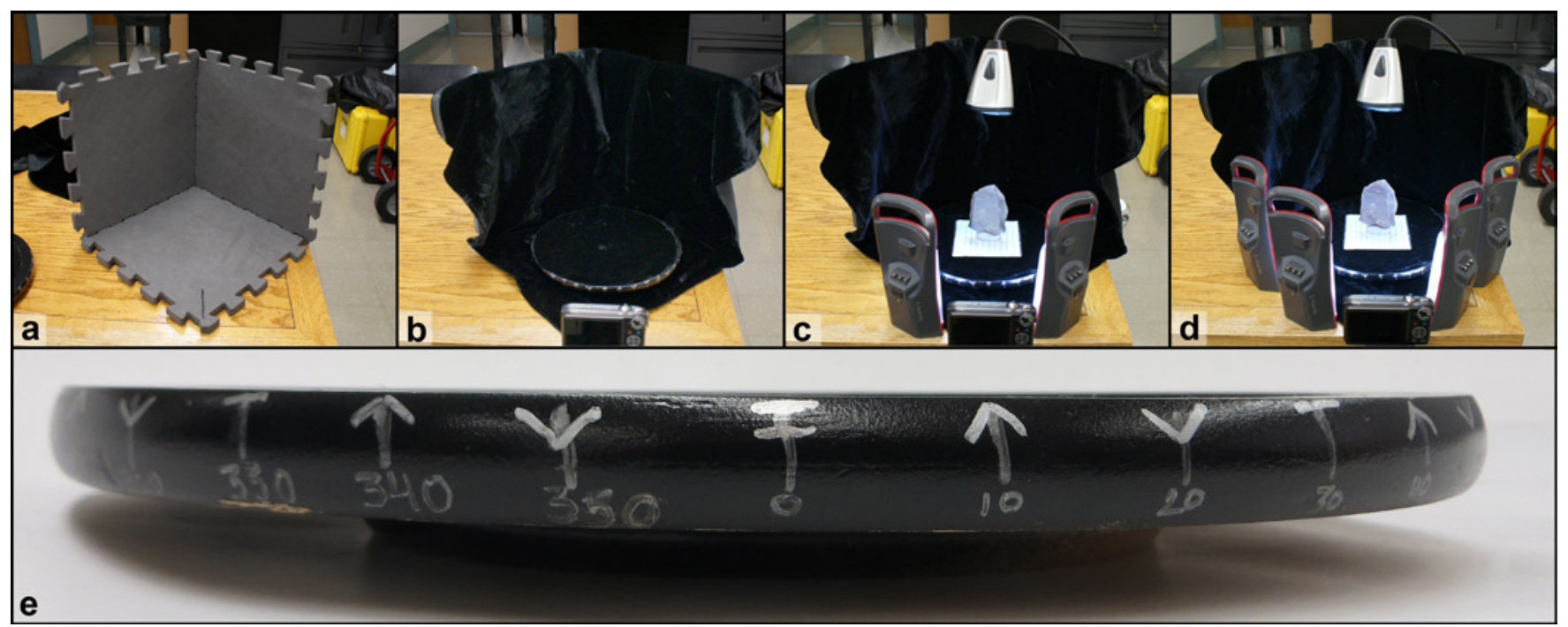

FIGURE 2. Setup and details of the portable photogrammetry rig described in this paper: (a) piecing together foam tiles; (b) addition of velvet backdrop, turntable covered with velvet, camera, and tripod; (c) addition of artifact, scale, overhead lamp, and two panel lamps; (d) four lamp version of the setup used when more side illumination is required; (e) detail of the turntable edge showing, $T, \uparrow$, and Y-shaped symbols used to denote object orientations to be captured during the first, second, and third rotations of photo-taking.

$30^{\circ}$ so that the $50^{\circ}$ mark is facing the camera. Take another photo. Continue rotating the turntable and taking photographs every $30^{\circ}$ until the turntable has been fully rotated (i.e., at the $80^{\circ}, 110^{\circ}, 140^{\circ}, 170^{\circ}, 200^{\circ}, 230^{\circ}, 260^{\circ}, 290^{\circ}, 320^{\circ}$, and $350^{\circ}$ marks). On our turntable, these intervals are annotated with a Y-shaped symbol.

7. At this point, you should have taken 37 photos total: one of the object label and 36 of the object.

8. Depending on the shape of the object, it may be necessary to raise and tilt the camera once again and take an additional round of photographs in order to sufficiently capture the top of the object. Usually, we take fewer photographs at this camera position (e.g., four photos with the turntable at the $0^{\circ}, 90^{\circ}, 180^{\circ}$, and $270^{\circ}$ positions.

9. Remove the target object and take a photo of the scale alone. This marks the transition between sets of object photos and will help organize photos later on.

10. Turn the object over, flipping it $180^{\circ}$. What was previously the top of the object should now be facing downward towards the turntable's surface.

11. Repeat the photography protocol as before, but in reverse. In other words, begin taking photos with the camera in a very high position and work downward systematically rotating the object as before.

\section{Camera Settings}

There are several guides to best practices for photography for close-range photogrammetry available on the web (e.g., Barnes 2011; Cultural Heritage Imaging 2014). In line with these suggestions, we adopted the following practices during the data acquisition phase of this project.

\begin{tabular}{l|} 
CHI citation should \\
be for 2015
\end{tabular}

Manual camera settings were always used. Specifically, for the models presented later in this article, the International Organization for Standardization (ISO) setting (i.e., the sensitivity of the camera's light sensor) was set as low as possible on our camera (80) in order to minimize the amount of noise in each image. Secondly, we avoided low F-stops, since they tend to result in a shallow depth of field, which can leave part of the target object out of focus. For the models presented in this article, we used our camera's maximum F-stop of 8. Because of the low ISO and high F-stop employed, a shutter speed of either one-thirteenth or one-fifteenth of a second was generally required. We note that, when using these shutter speeds, a tripod is essential for capturing clear images. Finally, we set the camera on a two-second delay in order to avoid blurriness that can result from movement caused by pressing the shutter button on the camera body.

\section{THE LES COTTÉS LITHIC SAMPLE}

Les Cottés is a cave site located at southwestern margins of the Parisian basin (France). Its archaeological deposits were originally discovered at the end of the nineteenth century. Modern excavations were initiated at the site in 2006 and are still ongoing (Soressi et al. 2010). The Les Cottés sequence has yielded five main archaeological layers, encompassing the final Mousterian through the Early Aurignacian (Roussel and Soressi 2013). The sequence is 1.5 to $4 \mathrm{~m}$ thick and corresponds to a tight chronological window of 35,080 cal B.P. to 45,800 cal B.P. (Jacobs et al. 2015; Talamo et al. 2012). Les Cottés is especially important to the study of the Middle to Upper Paleolithic Transition in Western Europe as it is one of a few rare sites that contains layers of distinct lithic industries associated with both the last Neanderthals and the first anatomically modern humans in the region (i.e., the Châtelperronian and the Protoaurignacian, respectively). Moreover, these industries are separated by very 


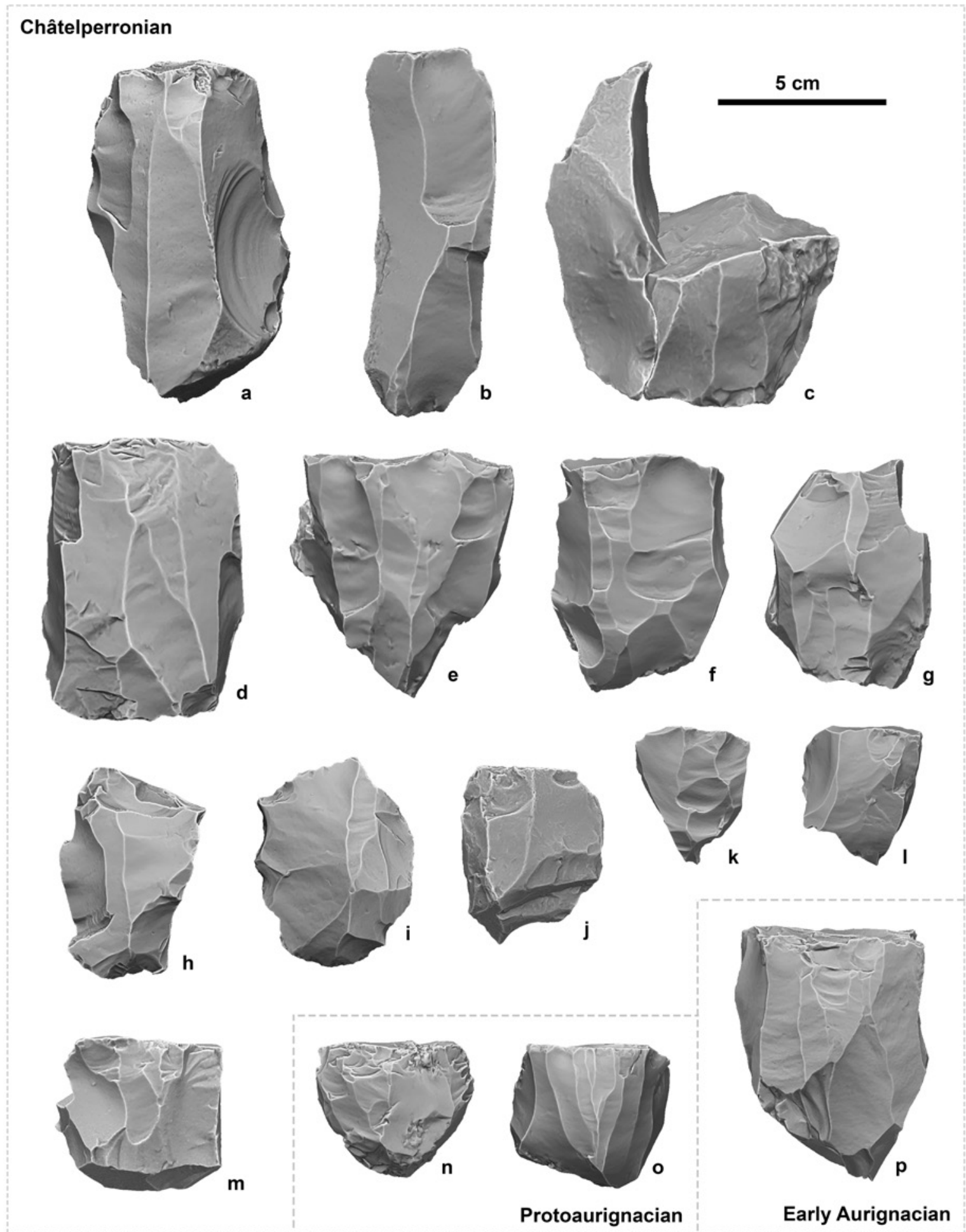

FIGURE 3. Models of lithic cores from Les Cottés displayed using "curvature mode" in Geomagic Design X to highlight model details. This mode displays curvature as a spectrum of grays. More convex areas are lighter gray while more concave areas are darker; (a) R5-1485; (b) Y4-390; (c) refit between Y4-313 (removal) and X6-106 (core); (d) Y4-2021; (e) Y4-854; (f) Y6-1574; (g) Y5-2500; (h) Y5-2865; (i) Y5-2740; (j) Y5-2612; (k) Y4-866; (l) Z4-1201; (m) Y4-294; (n) S6-939; (o) S6-993; (p) Z6-851. Additional information about each artifact is available in Supplemental Table 1. 
low density or sterile layers, making the common problem of stratigraphic mixing unlikely (Soressi et al. 2010)

Over the 2014 excavation season, 15 cores and one bladeto-core refit were captured using the photogrammetry rig described above. The cores in this sample range from 4.2 to 10.7 $\mathrm{cm}$ in their largest dimension and include several different raw materials of differential quality and composition. Artifacts were chosen from the Châtelperronian, Protoaurignacian, and Early Aurignacian in order to showcase a variety Upper Paleolithic core types, including some atypical examples. These models were created for several reasons. First, they serve as a portable and diffusible digital reference collection. We believe that this is particularly important for archaeological materials that are relatively rare, as is the case for the Les Cottés Châtelperronian material. Second, these 3D models have the potential to be used in future studies employing techniques such as geometric morphometrics. Finally, the models used here demonstrate the quality that can be produced using a photogrammetry rig system, as described in this paper. Two-dimensional representations of all the completed 3D models created in 2014 are shown in Figure 3. Three manipulatable 3D models have also been embedded in this paper (Figures 4a-c). A complete collection of 3D artifact models may be download in the form of 3D PDFs or OBJs as part of this paper's supplemental materials (Supplemental 3D PDFs 1 through 16).

\section{THE USE OF DEVELOPER SPRAY}

Shiny, translucent, and overly homogeneous materials can be difficult to capture in a 3D format. This is true not just for photogrammetry, but also for laser and structured light scanning. A large portion of the lithic artifacts from Les Cottés are made of raw materials that fit this description. Dusting an object with an inert mineral powder, often in the form of developer spray was found to mitigate many of the issues caused by these material characteristics, in terms of both 3D scanning and more traditional practices such as artifact photography and illustration (Airvaux 2005). This spray comes in an aerosol can and consists of talc particles suspended in an alcohol and acetone based solution, which acts as a drying agent. It is normally used as part of a multi-stage process to check the quality of welds in industrial settings or to highlight imperfections in automotive finishes. In Europe, the product used was Révélateur Skincric R. 764 S1. In the United States, an equivalent product is Magnaflux SKD S2 Developer.

It was hypothesized that developer spray would be effective in improving the quality of models produced on difficult raw materials not only because the spray would reduce shine, but, more importantly, because it would create a much more detailed pattern of reference points than one would have otherwise. This, in turn, would give the photogrammetry software additional data to use in computing object morphology. This is demonstrated in Figures $5 a$ and $5 b$.

To apply the spray, the laminated scale was used as a rigid base to hold and rotate the object (Figure 5c). To avoid unnecessary mess, both the scale and kneadable eraser used to support the object were protected using plastic kitchen wrap. Since the spray powder comes off with any direct handling, it must be washed off and reapplied before an object can be flipped to capture it in its second orientation. Cortical areas of objects, as well as those with a pre-existing complex pattern of patina, were not coated. The spray may be cleaned off of most non-porous lithic materials, using water and a soft bristled toothbrush or sponge. The use of this type of spray on fragile or porous objects that cannot easily be washed, or on artifacts that may have traces of ancient residues, would obviously not be recommended. Also, since the developer spray contains acetone, some types of artifact labeling may be adversely affected.

In order to test the effectiveness of developer spray in augmenting the quality of 3D models produced with Photogrammetry, several models of core Y6-2021 were created using sets of photos taken both with and without a coating of developer spray and were run using both medium- and high-quality settings in PhotoScan for the "build dense cloud" stage of processing. The core is mostly unpatinated and is comprised of a shiny, semi-translucent raw material (see details in Supplemental Table 1). As anticipated, the uncoated artifact produced a $3 \mathrm{D}$ model that conforms to the overall morphology of the real artifact but lacks details that are important for lithic analysis, such as ripples, which are indicative of the direction of flake removal (Figure 6). Furthermore, the model's surface appears unrealistically rough. Running the same set of photos of the uncoated artifact on the high-quality setting in PhotoScan at the "build dense cloud stage" did not produce a superior model, but only added to the amount of "noise" on the object's surface. ${ }^{9}$ The use of developer spay both decreased the amount of noise and increased the amount of detail visible on the final digital model. This is noticeable even at the medium-quality level of processing. The same set of photos with developer spray run on high revealed even more detail, including minute differences in the graininess of the raw material and ripples indicative of scar directionality.

As seen in this example, one major negative aspect of using developer spray is that it obscures details of the object's surface, including color, transparency, and, in the case of lithic artifacts, internal fracturing. This means that the resulting model texture wraps (i.e., the digital images applied to the model's surfaces) do not correspond to what the artifacts look like in real life. ${ }^{10}$ It is also important to note that rougher models created without using developer spray can be made to look better by adjusting render settings. This flat rendering style is devoid of artificial shadows and highlights. Models displayed this way both hide mesh defects and have the advantage of having true-to-life texture wraps (see the bottom right model in Figure 6). ${ }^{11}$ As a result, the use of developer spray may not always be advisable for applications for which the overall look of the finished model is more important than the amount of fine morphological detail in the model itself, for example when creating models for the web or an exhibit intended for the general public.

Since accurately capturing artifact morphology was the primary goal of this particular project, the majority of the remaining cores in the sample were coated with developer spray prior to being photographed. Exceptions included a core comprised of a matte raw material (Y6-2612) and artifacts with already complex surface texture due to raw material inclusions and/or patina (Y4-313 and X6-106). Artifacts that were partially patinated were sprayed only on their unpatinated surfaces. 

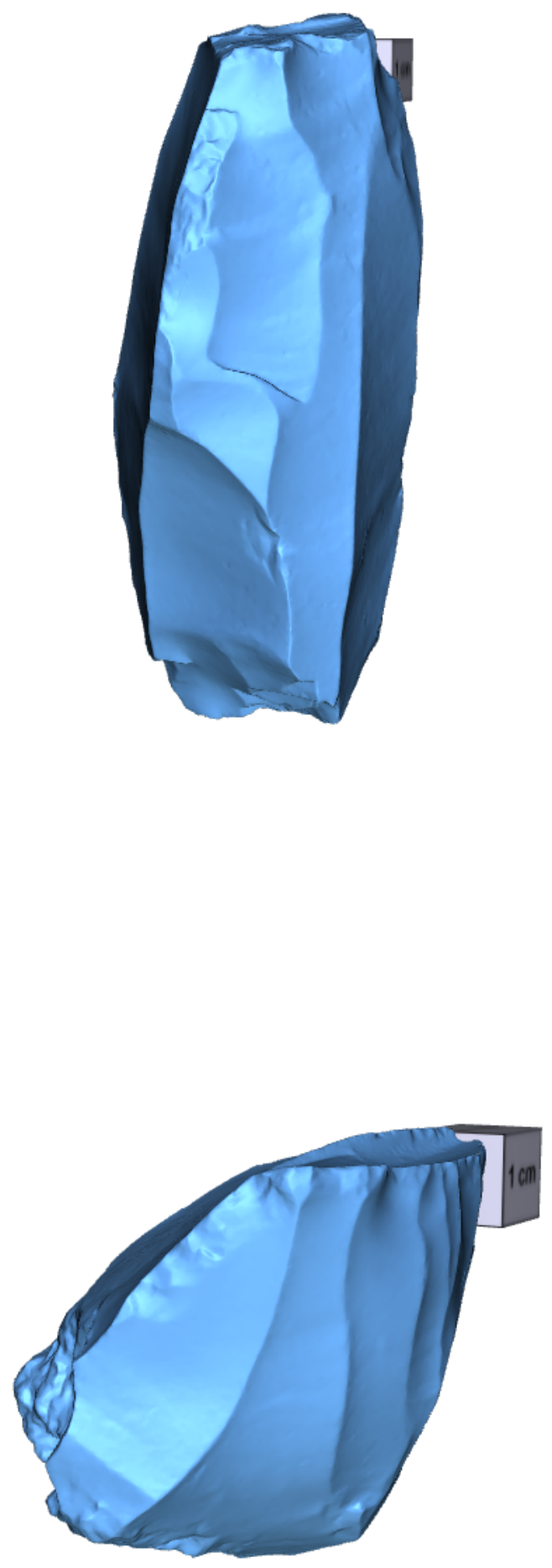


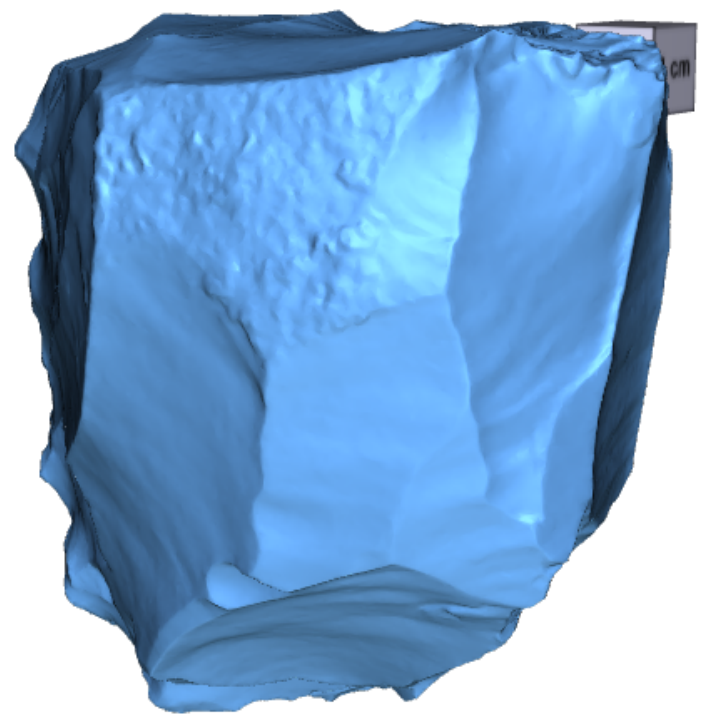

FIGURE 4. Three 3D models of cores from three different lithic industries found at Les Cottés: (a) Châtelperronian core R51485; (b) Protoaurignacian core S6-993; (c) Early Aurignacian core Z6-851. Models have been reduced to 150,000 polygons to limit file size.

\section{Model Processing}

The core models created for this paper were processed using a combination of Agisoft PhotoScan Standard Edition and Geomagic Design X.12 This was due to the software resources available to us at the time. Geomagic was used to compensate for features that are lacking in the Standard Edition of Agisoft PhotoScan but are available in the Professional Edition. One important feature that the standard edition of PhotoScan lacks is the ability for users to manually place reference markers on their models. Markers can be used to manually align models of two different sides of an object together in the event that automatic alignment fails, as well as to define model scale.

Although Geomagic is very powerful, licenses for the software are relatively expensive. ${ }^{13}$ For those who do not already have access to the program, using an open source 3D modeling application such as Meshlab, or simply purchasing Agisoft PhotoScan Professional Edition, would likely be a better solution.

\section{CLOSE-RANGE PHOTOGRAMMETRY COMPARED TO OTHER METHODS OF 3D SCANNING}

What is the best method of 3D scanning? In short, there is no universal answer. There are a multitude of factors that contribute to the quality of scan that can be produced using different methods, on different objects, in different environments. In this section, we consider three major factors that workers may take into account when choosing a 3D scanning method, and highlight situations in which close-range photogrammetry may be the most viable option.

\section{Cost}

There are numerous types of 3D scanners currently available on the market at a wide array of price points. The cost of 3D 

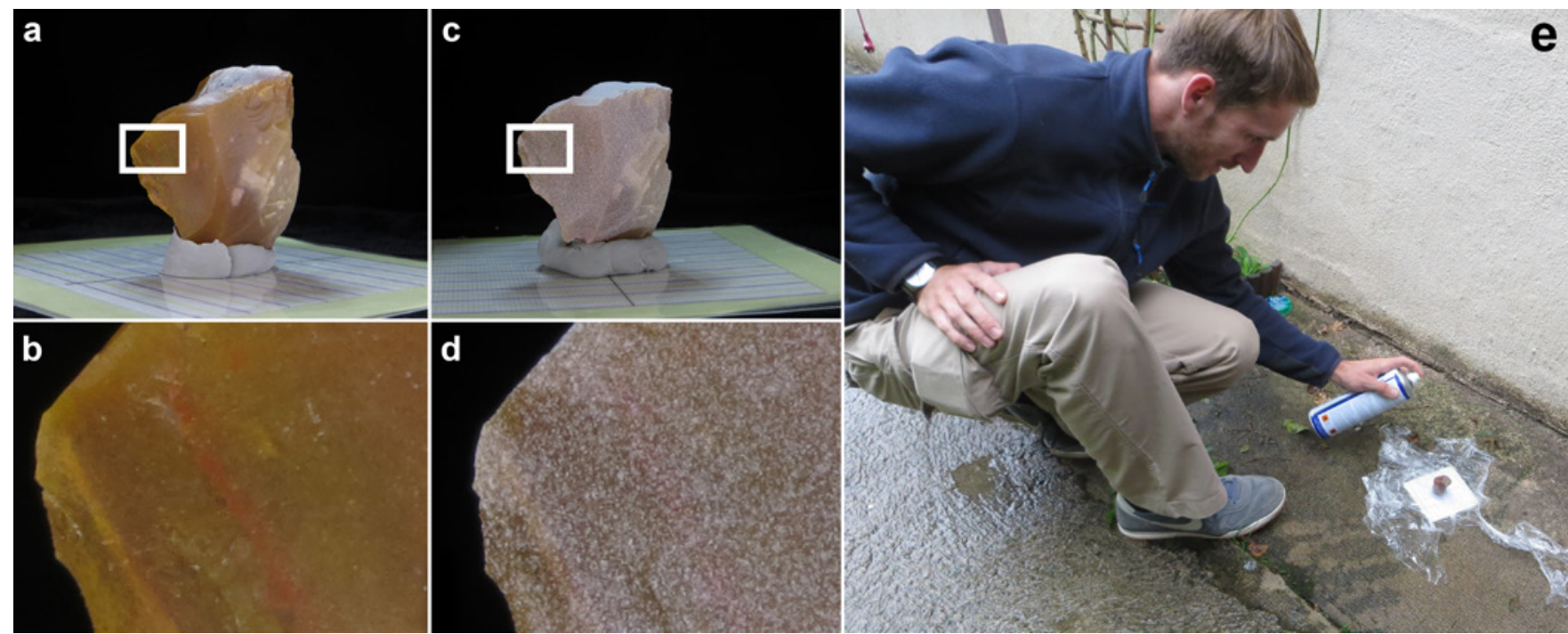

FIGURE 5. The use of talc developer spray to improve photogrammetric model quality: (a) Z4-1201 without developer spray; (b) section of above photo enlarged eight times to show detail; (c) Z4-1201 with an appropriate amount of developer spray applied; (d) above photo enlarged eight times to show detail; (e) developer spray being applied in the field.

scanners most often employed by archaeologists currently tends to fall within the range of approximately $\$ 3,000$ (see Table 1). In comparison, excluding the camera and tripod, which most archaeologists will already have access to, the combined cost of the rig elements described in this paper comes out to less than $\$ 125$. The price of photogrammetry software ranges from free for open source options to several thousands of dollars for a professional license of Agisoft PhotoScan for non-educational users. That being said, for educational users, the combined cost of a Professional license of Agisoft PhotoScan, plus all of the other photogrammetry rig elements we list, is still significantly less than the cost of most 3D scanners. Since photogrammetry software can also be used to model things at larger scales, such as excavation features, sites, and even landscapes, it can be considered to have additional utility.

\section{Portability and Adaptability}

All of the rig elements described in this paper can be carried in a single carry-on-sized suitcase. In our experience, the cases used to transport both NextEngine laser and DAVID structured light scanners tend to fall into the size range of bags that must be checked in to fly. For projects for which the collection of $3 \mathrm{D}$ data is not the main goal, this amount of luggage could be prohibitive for an individual to transport, especially if other necessary but bulky field gear, such as camping equipment, is required.

Additionally, no components of this rig require an external source of power to operate. This means that the rig can be used in essentially any environment. A final point is that this type of photogrammetry setup does not contain any technological elements that have a high probability of breaking down in adverse conditions. Furthermore, many rig components are commercially available and can be replaced by users directly without too much hassle. This is not the case for other types of scanners, which may need to be sent back to the manufacturer for repair if individual parts break down. In a field situation, such an occurrence has the potential to shut down the $3 \mathrm{D}$ documentation element of a project completely.

\section{Time Investment}

The amount of time it takes to complete a full object scan can be divided into two phases. First, there is the time needed to collect the raw data. Second, there is the time it takes to process, align, and edit these data. For both laser and structured light scanners, these two phases sometimes occur simultaneously. In close-range photogrammetry, these phases are more distinct. Furthermore, the time it takes to process image data into a complete 3D model can vary greatly depending on image quality, the amount of detail sought, and the power of the computer being used. As a result, it is hard to directly compare the amount of time it takes to produce a finished object model using a laser, structured light, or photogrammetric solution. ${ }^{14}$

What can be said is that, in terms of raw data acquisition, photogrammetry is a relatively fast option. Once the authors of this paper became proficient in using this rig, both sides of an object could be photographed at a steady but judicious pace in a total of approximately 12 minutes on average. In the authors' experience, it is feasible for an individual to photograph at least 20 artifacts a day using this method. By comparison, Ahmed et al. (2014) state that their structured light system could usually scan a single object in less than 20 minutes. NextEngine scanners slower still. Magnani (2014) states that it took approximately 30 minutes to capture one orientation of an object and that at least two orientations had to be captured in order to obtain a complete object scan. The reduced amount of time that is required to collect the raw data necessary to produce photogrammetric models could be quite advantageous in situations in which collection access is very limited. 


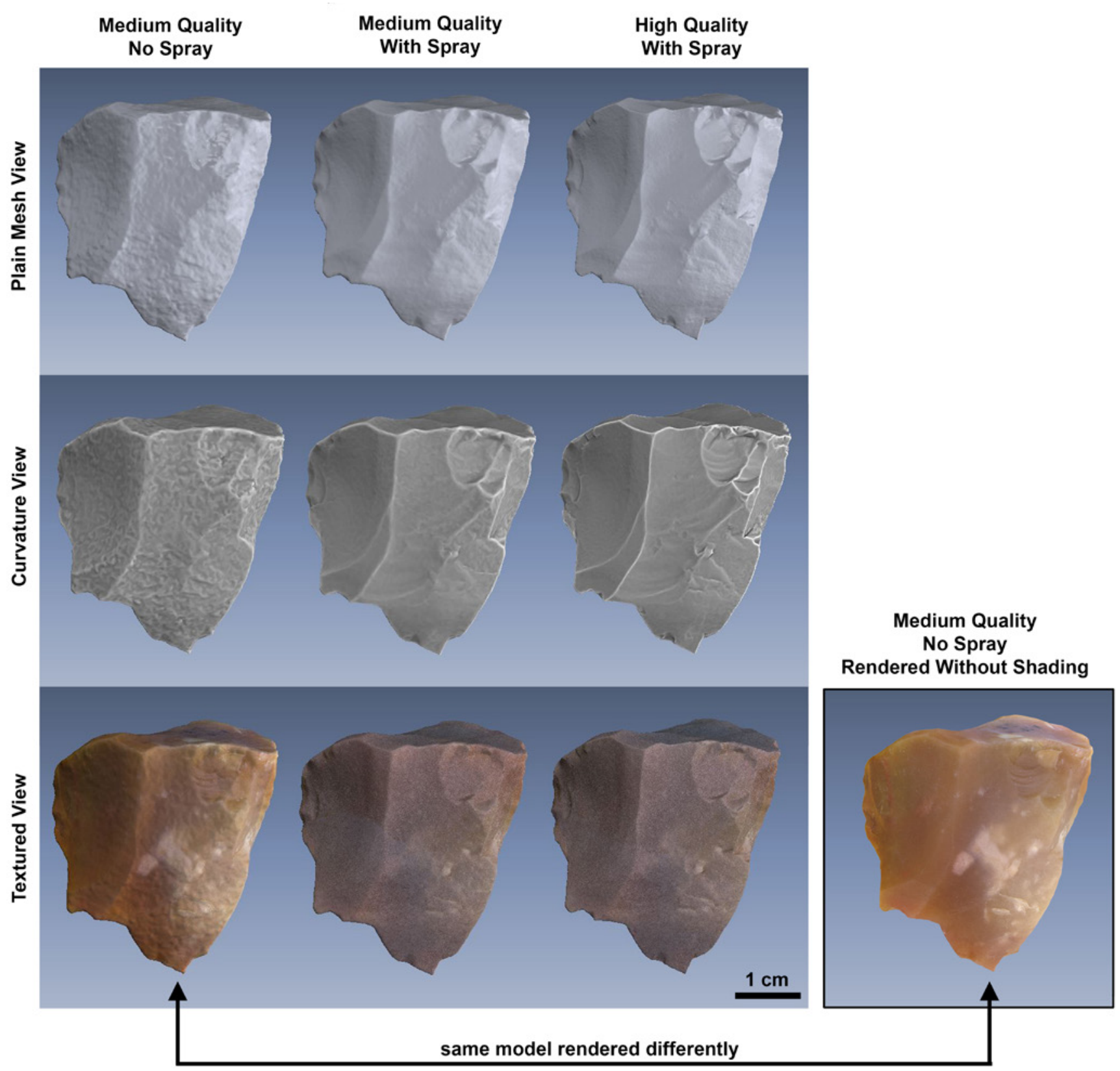

FIGURE 6. Different 3D models of Z4-1201 produced without and with talc spray and processed in Agisoft PhotoScan at different quality levels. The model shown in the far lower right is the same as the one shown in the leftmost column but has been rendered without shading and highlights from a virtual light source.

\section{Model Quality}

Several recent comparative studies have demonstrated that the gap in scan quality between models produced with close-range photogrammetry and those produced using other scanning methods is closing (e.g., Katz and Friess 2014; Kersten and Lindstaedt 2012; Koutsoudis et al. 2013). As stated previously, an almost innumerable number of elements contribute to the quality of 3D models obtained using different scanning methods, and it is impossible to make blanket statements regarding the superiority of one technique over another. Nevertheless, it is possible to remark on general trends.
In order to illustrate some of these trends, we present a comparison of three models of the same experimentally-produced lithic blade (Figure 7). This blade was knapped from an unspecified French chert, which happens to be particularly well suited to close-range photogrammetry due to its opacity and heterogeneity. The object was not coated in developer spray. The first scan was taken using a NextEngine laser scanner. The second model was produced using the photography rig and protocol described in this paper. The third model was created using a DAVID SLS-2 structured light scanner. In each case, some minor 


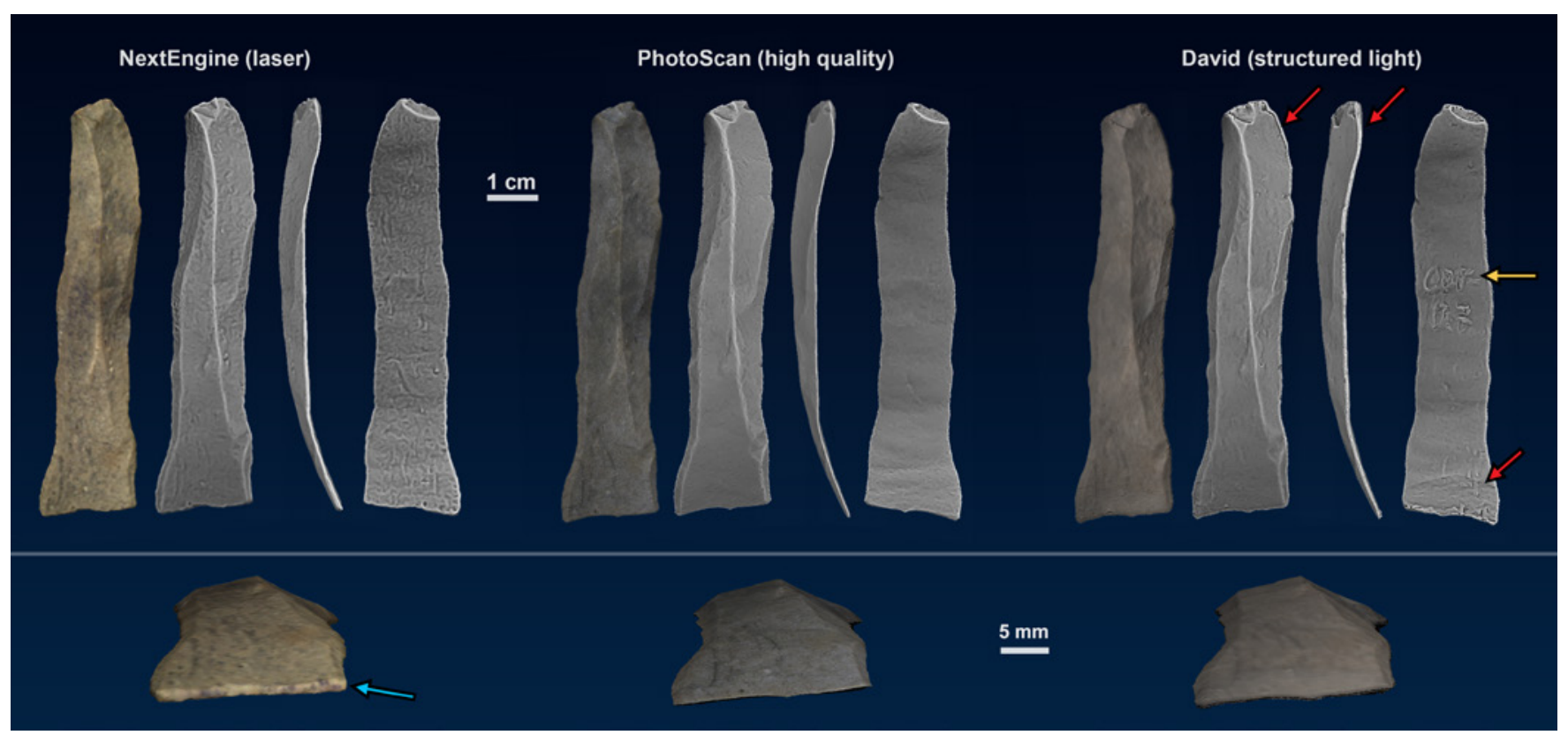

FIGURE 7. Comparison of scans of the same object produced with a NextEngine laser scanner, through image-based modeling in Agisoft PhotoScan, and with a DAVID structured light scanner. The blue arrow points to blunting on the piece's distal end on the laser scanned model due to missing data. The red arrows point to errors in the structured light model caused by improper scan alignment. The yellow arrow points to an error caused by the structured light scanner's misinterpretation of dark labeling on the object's surface.

editing, including filling holes in the mesh, was performed in Geomagic Design X.

First, we observe that the NextEngine produced the lowestquality scan both in terms of textural quality and in terms of the amount of noise that is visible on the scan's surface. Furthermore, the NextEngine did the worst job at capturing the blade's thin edges.

Although the DAVID structured light scanner was able to capture details on the dorsal face of the blade, it still had trouble modeling the object's edges. In order to produce complete object models, both laser and structured light scanners require multiple individual scans to be aligned and then merged together. In this case, improper alignment between these individual scans has resulted in an unnaturally bulbous edge on the proximal end of the piece and the appearance of features on the blade's extremely thin distal end that are not present on the actual object. Although these issues could be mitigated to some degree through manual alignment of individual scans, this process has the potential to be very time consuming and cannot make up for holes in the original scan data. This model also demonstrates the way in which structured light scanning has a tendency to erroneously misinterpret very light or dark areas, such as artifact labeling, as being three-dimensional features rather than being flat.

In this particular case, the photogrammetric model turned out to be the most accurate morphologically. In addition, compared to the other scans, the model's texture is the correct color and contains a much higher level of detail than the other scans. High-resolution photorealistic textures of this nature also have analytical utility. Features that are difficult, if not impossible, to capture in three dimensions using any scanning methods, such as fine retouch or lancets, can still be recognized on photogrammetric models (Figure 8 ). Although it is possible to overlay detailed photo-realistic textures to models acquired with laser or structured light scanners, the most common ways to achieve this are manual in nature and may be too time consuming to apply to large data sets (Ahmed et al. 2014).

\section{DISCUSSION}

\section{Applications of 3D Modeling}

Like many papers on the subject of 3D artifact documentation, this project speaks to issues of archaeological access in several of its forms. First of all, it addresses issues of researchers' access to collections. As has long been lamented, one major hurdle that archaeologists often have to overcome is the long distance between their field sites or the locations of the collections they are studying and their home base location. It is not out of the ordinary for researchers to live in different cities, states, or even continents from the material with which they are working. As a result, the ability to reference complex artifact morphologies at a later date in three dimensions can be extremely advantageous. As mentioned earlier, this is one of the main reasons we initially sought to digitize the materials from Les Cottés.

Problems of access are exacerbated when field-wide debates develop based on materials from a small number of sites whose collections are easily accessible to only a small number of researchers. This could be said to be the case for the study of the Châtelperronian industry, which is a principal focal point in the study and Neanderthal and anatomically modern human 

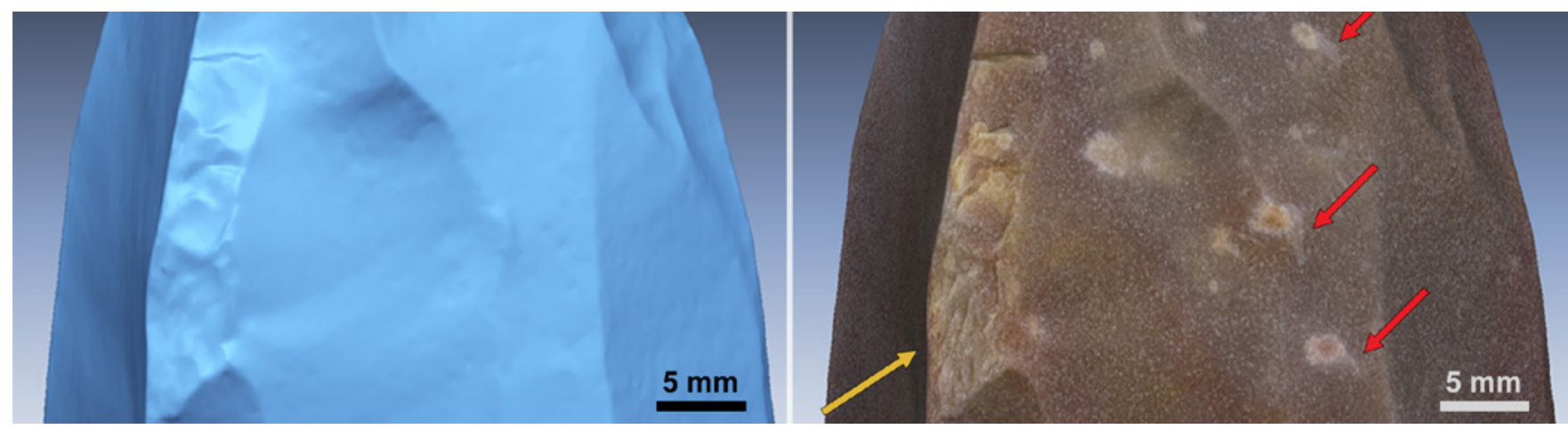

FIGURE 8. Details observable on the texture of a 3D model produced using photogrammetry that are not visible on the 3D mesh itself. The yellow arrow points to cresting removals that are similar in morphology to retouch. The red arrows point to lancets. Object shown is R5-1485.

interactions in Europe during the Middle to Upper Paleolithic transition (Soressi and Roussel 2014). The creation and publication of 3D models of artifacts gives researchers the ability to share this type of artifact data across sites, institutions, and borders in ways that were previously impossible.

3D models can also be used to display manually refit pieces (see Supplemental 3D Model 4 for an example). Individual removals may be hidden one at a time, making it possible to visualize in three dimensions a refit sequence from start to finish in a single interactive file. One problem lithic analysts sometimes run into is that particularly nice, and thus illustrative, examples of refit sequences are often glued or otherwise secured together for display. This makes it essentially impossible for further research to be conducted on the internal pieces without either dismantling the display or using techniques such as micro-CT (Abel et al. 2011). Digitizing artifacts and then refitting them in digital space can mitigate these issues.

In addition, the ability to spin and manipulate an object in digital 3D space can allow for a much more visceral comprehension of complex elements of object morphology compared to static $2 \mathrm{D}$ representations. Lithic cores were specifically targeted for this project for this reason. At the same time, the authors would agree with the position that, despite recent advances (Olson et al. 2014; Richardson et al. 2014), at least for the time being 3D models of lithic artifacts are best seen as being supplemental to traditional methods of recording and representation for publication rather than serving as true replacements. That being said, we would argue that our results demonstrate that photogrammetric models of lithic artifacts do indeed have the potential to be used for some illustrative purposes (contra Magnani 2014).

\section{CONCLUSION}

The documentation of artifacts Acknowledgement an increasingly accessible and added / slight re-wording ties in a wide variety of contexts, including object analysis, artifact publication, and the sharing of raw data. As a method of $3 \mathrm{D}$ registration, photogrammetry has the benefits of being affordable, reliable, and better adapted for use in adverse field conditions and when collection access time in limited. For these reasons, it has the potential to make the practice of $3 \mathrm{D}$ data col- lection, analysis, and dissemination possible for a whole new set of people from private collectors to students and professional archaeologists. This paper has introduced a portable photogrammetry rig that increases the efficiency of photo capturing and model processing. Through a combination of techniques, including systematic and precise photography and the use of developer spray, we were able to reliably produce highly detailed 3D models.

Recent improvements in methods, software, and computing power have begun to shrink the gaps between the quality of model it is possible to produce using photogrammetry and the quality of other scanning methods. It is hoped that further work in the field will be able to continue to evaluate the quality of photogrammetric models quantitatively. This includes further statistically assessing the accuracy of 3D models and various measurements taken from them in comparison to their analog counterparts. Studies of this type will remain essential in giving researchers the ability to determine the most appropriate applications for the use of one 3D digitization method over another across disciplines as technology advances.

Lastly, we would like to advocate for more communication between computer scientists, imaging experts, and all archaeologists, including those who are less technically focused. The creation of an expanded library of literature that is in dialog with both the technical and practical sides of archaeology has the potential to raise the level of practice across the entire discipline.

\section{Acknowledgments}

Should be spelled

We thank Glen Favretto, Kristina Golubiewski-Davis, Matt Hunstiger, Steffen Schatz, Kele Missel, Eduard Pop, and Gilbert Tostevin for their contributions to various aspects of this project. We would also like to thank the editorial staff of Advances in Archaeological Practice, as well as this paper's anonymous reviewers, for their helpful comments. The project is funded by the Max Plank Society (Germany) and the

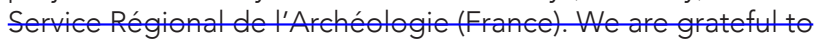
Jacques Bachelier, owner of the site, for access to his property and to Jean-Jacques Hublin, director of the Department of Human Evolution at the MPI EVA (Leipzig, Germany), for scien tific and financial support. Samantha Porter's research has been 
funded by a Thomas F. Wallace Fellowship from the University of Minnesota and a grant from the Leakey Foundation.

\section{Data Availability Statement}

Supplemental materials associated with this article are conserved on the online Data Repository for the University of Minnesota (DRUM). They may be accessed at the following persistent address: http://dx.doi.org/10.13020/D6201D. These materials include 3D PDF files of all the presented cores from Les Cottés, as well as additional information regarding their archaeological context. Two tutorials describing automatic background masking and the masking of supporting from model textures in Agisoft PhotoScan may also be downloaded from DRUM, along with a printable version of the newest photogrammetric scale we have developed. Access is also available upon request to $O B J$ versions of the $3 D$ core models for teaching and research purposes via DRUM. Technical questions about photogrammetry methods may be directed towards S. Porter (port0228@umn.edu). Questions regarding the Les Cottés site and materials may be directed towards M. Soressi (m.a.soressi@ arch.leidenuniv.nl). Questions about the lithic technology of the artifacts presented here may be directed towards M. Roussel (m.b.roussel@arch.leidenuniv.nl).

\section{Supplemental Materials}

Supplemental materials accompanying this article are available online through DRUM at http://dx.doi.org/10.13020/D6201D:

Supplemental Table 1. Additional information about the archaeological context of artifacts and processing of models presented in this paper.

Supplemental 3D PDFs. 3D PDF files of the Les Cottés artifact models presented in this paper. Each PDF file includes versions of the model both with and without texture.

Supplemental Model OBJs. Editable OBJ files of the Les Cottés artifact models presented in this paper. Access to these files for educational and research purposes will granted be on request.

Supplemental Instructions 1. Background masking tutorial reviewing how to automatically mask images with a black background in Agisoft PhotoScan using two different methods.

Supplemental Instructions 2: Masking artifact support tutorial explaining how to mask artifact support elements, such as putty, from object images so that supporting elements do not appear as part of a finished model's texture.

Supplemental Scale 1. This photogrammetric scale may be placed under objects during image acquisition to facilitate image alignment and model scaling.

A supplemental video is available online through the SAA member login at www.saa.org/member-login:

Supplemental Video 1. Video demonstrating our image capture protocol using our photogrammetry rig. Click video to play.

\section{REFERENCES CITED}

Abel, Richard L., Simon Parfitt, Nick Ashton, Simon G. Lewis, Beccy Scott, and Chris Stringer

2011 Digital Preservation and Dissemination of Ancient Lithic Technology with Modern Micro-CT. Computers and Graphics 35:878-884.

Ahmed, Namir, Michael Carter, and Neal Ferris

2014 Sustainable Archaeology through Progressive Assembly 3D Digitization. World Archaeology 46:137-154.

Airvaux, Jean

2005 Note sur de nouveaux procédés pour le dessin des objets lithiques. Bulletin Préhistoire du Sud-Ouest 12:218-221.

Barnes, Adam

2011 Close-Range Photogrammetry: A Guide to Good Practice in Archaeology Data Service / Digital Antiquity Guides to Good Practice. Archaeology Data Service, University of York, UK. Electronic document, http://guides.archaeologydataservice.ac.uk/g2gp/Photogram Toc, accessed June 6, 2015.

Blais, François

2004 Review of 20 Years of Range Sensor Development. Journal of Electronic Imaging 13:231-240.

Bretzke, Knut, and Nicholas J. Conard

2012 Evaluating Morphological Variability in Lithic Assemblages Using 3D Models of Stone Artifacts. Journal of Archaeological Science 39:3741-3749.

Clarkson, Chris

2013 Measuring Core Reduction Using 3D Flake Scar Density: A Test Case of Changing Core Reduction at Klasies River Mouth, South Africa. Journal of Archaeological Science 40:4348-4357.

Clarkson, Chris, and Peter Hiscock

2011 Estimating Original Flake Mass from 3D Scans of Platform Area. Journal of Archaeological Science 38:1062-1968.

Clarkson, Chris, Lucio Vinicius, and Marta Mirazón Lahr

2006 Quantifying Flake Scar Patterning on Cores Using 3D Recording Techniques. Journal of Archaeological Science 33:132-142.

Cultural Heritage Imaging

2014 Photogrammetry. Electronic document, http:// culturalheritageimaging.org/Technologies/Photogrammetry/ June 6, 2015

$\mathrm{CHI}$ citation

vis, Lorten G., Daniel W. Bean, Alex J. Nyers, and David R. Brauner 2015 GLIMR: A GIS-Based Method for the Geometric Morphometric Analysis of Artifacts. Lithic Technology 40: 199-217.

Gingerich, Joseph A. M., Sabrina B. Sholts, and Sebastian K. T. S. Wärmländer 2014 Fluted Point Manufacture in Eastern North America: An Assessment of Form and Technology Using Traditional Metrics and 3D Digital Morphometrics. World Archaeology 46:101-122.

Grosman, Leore, Gonen Sharon, Talia Goldman-Neuman, Oded Sikt, and Uzy Smilansky

2011 Studying Post Depositional Damage on Acheulian Bifaces Using 3-D Scanning. Journal of Human Evolution 60:398-406.

Grosman, Leore, Oded Smikt, and Uzy Smilansky

2008 On the Application of 3-D Scanning Technology for the Documentation and Typology of Lithic Artifacts. Journal of Archaeological Science 35:3101-3110.

Jacobs, Zenobia, Bo Li, Nathan Jankowski, and Marie Soressi

2015 Testing of a Single Grain OSL Chronology across the Middle to Upper Palaeolithic Transition at Les Cottés (France). Journal of Archaeological Science 54:110-122.

Katz, David, and Martin Friess

2014 Technical Note: 3D from Standard Digital Photography of Human

Crania-A Preliminary Assessment. American Journal of Physical

Anthropology 154:152-158.

Kersten, Thomas P., and Maren Lindstaedt 
2012 Image-Based Low-Cost System for Automatic 3D Recording and Modelling of Archaeological Finds and Objects. In Progress in Cultural Heritage Preservation. 4th International Conference, EuroMed 2012, Limassol, Cyprus, October 29 - November 3, 2012 Proceedings, edited by Marinos loannides, Dieter Fritsch, Johanna Leissner, Rob Davies, Fabio Remondino, and Rossella Caffo, pp. 1-10. Springer, Berlin.

Koutsoudis, Anestis, Blaž Vidmarb, and Fotis Arnaoutoglou

2013 Performance Evaluation of a Multi-image 3D Reconstruction Software on a Low-Feature Artefact. Journal of Archaeological Science 40:4450-4445.

Lin, Sam C., Matthew J. Douglass, Simon H. Holdaway, and Bruce Floyd

2010 The Application of 3D Laser Scanning Technology to the Assessment of Ordinal and Mechanical Cortex Quantification in Lithic Analysis. Journal of Archaeological Science 37:694-702.

Lin, Sam C., Zeljko Rezek, David Braun, and Harold L. Dibble

2013 On the Utility and Economization of Unretouched Flakes: The Effects of Exterior Platform Angle and Platform Depth. American Antiquity 78:724-745.

Luhmann, Thomas, Stuart Robson, Stephen Kype, and Jan Boehm

2013 Close-Range Photogrammetry and 3D Imaging. 2nd ed. Walter de Gruyter $\mathrm{GmbH}$, Berlin.

McCarthy, John

2014 Multi-Image Photogrammetry as a Practical Tool for Cultural Heritage

Survey and Community Engagement. Journal of Archaeological Science 43:175-185.

Magnani, Matthew

2014 Three-Dimensional Alternative to Lithic Illustration. Advances in Archaeological Practice 2:285-297.

Means, Bernard K., Ashley McCuistion, and Courtney Bowles

2013 Virtual Artifact Curation of the Historical Past and the NextEngine Desktop 3D Scanner. Technical Briefs in Historical Archaeology 6:1-12.

Morales, Juan I., Carlos Lorenzo, and Josep M. Vergès

2015 Measuring Retouch Intensity in Lithic Tools: A New Proposal Using 3D Scan Data. Journal of Archaeological Method and Theory 22:543-558.

Olson, Brandon R., Jody M. Gordon, Curtis Runnels, and Steve Chomyszak

2014 Experimental Three-Dimensional Printing of a Lower Paleolithic Handaxe: An Assessment of the Technology and Analytical Value. Lithic Technology 39(3):162-172.

Olson, Brandon R., Ryan A. Placchetti, Jamie Quartermaine, and Anne E. Killebrew

2013 The Tel Akko Total Archaeology Project (Akko, Israel): Assessing the Suitability of Multi-Scale 3D Field Recording in Archaeology. Journal of Field Archaeology 38:244-262.

Richardson, Eitan, Leore Grosman, Uzy Smilansky, and Michael Werman 2014 Extracting Scar and Ridge Features from 3D-scanned Lithic Artifacts. In Archaeology in the Digital Era: Papers from the 40th Annual Conference of Computer Applications and Quantitative Methods in Archaeology (CAA), Southampton, 26-29 March 2012, edited by Graeme Earl, Tim Sly, Angeliki Chrysanthi, Patricia Murrieta-Flores, Constantinos Papadopoulos, Iza Ramonowska, and David Wheatley, pp. 83-92. Amsterdam University Press, Amsterdam.

Riddle, Andrew T. R., and Michael Chazan

2014 Stone Tools from the Inside Out: Radial Point Distribution. World Archaeology. 46:123-136.

Roussel, Morgan, and Marie Soressi

2013 Une nouvelle séquence du Paléolithique supérieur ancien aux marges sud-ouest du Bassin parisien: Les Cottés dans la Vienne. In Le Paléolithique supérieur ancien de l'Europe du nord-ouest (35 000-15000 $B P$ ). Réflexions et synthèses à partir d'un Projet Collectif de Recherches sur le Paléolithique supérieur ancien du Bassin parisien, edited by Pierre Bodu, Lucile Chehmana, Laurent Klaric, Ludovic Mevel, Sylvain Soriano, and Nicolas Teyssandier, pp. 283-297. Mémoire LVI de la Société Préhistorique Française. Société Préhistorique Française, Paris.

Shott, Michael
2014 Digitizing Archaeology: A Subtle Revolution in Analysis. World Archaeology 46:1-9.

Shott, Michael J., and Brian W. Trail

2010 Exploring New Approaches to Lithic Analysis: Laser Scanning and Geometric Morphometrics. Lithic Technology 32:195-220.

Soressi, Marie, and Morgan Roussel

2014 European Middle to Upper Palaeolithic Transitional Industries:

Châtelperronian. In Encyclopedia of Global Archaeology, edited by Claire Smith, pp. 2679-2693. Springer, New York.

Soressi, Marie, Morgan Roussel, William Rendu, Jérôme Primault, Solange Rigaud, Jean-Pierre Texier, Daniel Richter, Sahra Talamo, Florian Ploquin, Blandine Larmignat, Carlotta Tavormina, and Jean-Jacques Hublin 2010 Les Cottés: nouveaux travaux sur l'un des gisements de référence pour la transition Paléolithique moyen-supérieur. In Préhistoire entre Vienne et Charente: Hommes et Sociétés du Paléolithique, edited by Buisson-Catil, Jacques and Jerôme Primault, pp.221-234. Association des Publications Chauvinoises, Chauvigny, France.

Sumner, T. Alexandra, and Andrew T. R. Riddle

2008 Virtual Paleolithic: Assays in Photogrammetric Three-Dimensional Artifact Modelling. PaleoAnthropology, 158-169.

Talamo, Sahra, Marie Soressi, Morgan Roussel, Mike Richards, and JeanJacques Hublin

2012 A Radiocarbon Chronology for the Complete Middle to Upper Palaeolithic Transitional Sequence of Les Cottés (France). Journal of Archaeological Science 39:175-183

\section{NOTES}

1. NextEngine Desktop 3D Scanners and accompanying software, ScanStudio HD, are produced by NextEngine, Inc.

2. Breuckmann smartSCAN white light scanners are produced by AICON and Breuckmann.

3. DAVID-SLS-2 scanners are produced by the DAVID group.

4. AgiSoft PhotoScan was developed by AgiSoft LLC.

5. VisualFSM was developed by Changchang Wu (http://ccwu.me/vsfm/).

6. PMVS 2 was developed by Yasutaka Furukawa (http://www.di.ens.fr/ cmvs/).

7. MeshLab was developed by the Visual Computing Lab ISTI-CNR.

8. 123D Catch was developed by Autodesk.

9. This model is not pictured in $\mathrm{x}$, but may be viewed in Supplemental 3D PDFs 15_d and 15b.

10. After data collection for this paper occurred, we established a workflow that makes it possible to overlay a realistic texture over a high-quality model generated from a sprayed object. This is done by taking two full sets of photos of the target object in the same position relative to the scale/base, one with developer spray and one without. Both sets of photos are aligned with one another. Photos with spray are used to construct model geometry, while photos without spray are used to build texture. We note that the increase in the number of photos required substantially increases the time necessary for both data collection and post-processing.

11. This unshaded rendering style is the default for textured 3D PDFs created within Agisoft PhotoScan. The textured 3D PDF models included in the supplemental materials for this paper are all rendered in this style, with the exception of the refit of Y4-313 and X6-106 (Supplemental 3D PDF 4).

12. Geomagic Design $X$ is produced by $3 D$ Systems.

13. Current prices for Geomagic Design $X$ are not publically listed.

14. Although we did not record model processing times for the Les Cottés material, model processing times were systematically recorded as part of another ongoing study. For this sample, high-quality models took an average of 2 hours and 13 minutes to process, while medium-quality models took an average 43 minutes. The majority of this time was taken up by the "build dense cloud" stage of model processing. User input is not required while this process is running. These listed times include all stages of post-processing, beginning with the masking of images in Adobe Photoshop and concluding with the scaling of finished models. 
The sample for this dataset includes 13 experimentally produced lithic artifacts, which were not coated in developer spray. Photos were taken with the same camera and photogrammetry rig that were used to capture the sample from Les Cottés. The same set of photos was used to produce both high- and medium-quality models. Models were processed in Agisoft PhotoScan Professional Edition on a computer with $32.0 \mathrm{~GB}$ of RAM and an Intel Xeon CPU E5-2630 v2 2.60 GHz processor. 